\title{
Noise-assisted classical adiabatic pumping in a symmetric periodic potential
}

\author{
O. Usmani ${ }^{1}$, E. Lutz ${ }^{1,2}$ and M. Büttiker ${ }^{1}$ \\ ${ }^{1}$ Département de Physique Théorique, Université de Genève 24, quai Ernest Ansermet CH-1211 Genève 4 \\ ${ }^{2}$ Sloane Physics Laboratory, Yale University, PO. Box 208120, New Haven, CT 06520-8120, USA
}

(November 3, 2018)

\begin{abstract}
We consider a classical overdamped Brownian particle moving in a symmetric periodic potential. We show that a net particle flow can be produced by adiabatically changing two external periodic potentials with a phase difference $\varphi$ in time and $\chi$ in space. The classical pumped current is found to be independent of the friction and to vanish both in the limit of low and high temperature. Below a critical temperature, adiabatic pumping appears to be more efficient than transport due to a constant external force.
\end{abstract}

PACS numbers: 05.40.-a, 05.40.Jc, 05.60.-k

\section{INTRODUCTION}

Recently there has been a considerable interest in small amplitude adiabatic pumping in mesoscopic electrical conductors [1]. Two oscillating, out-of-phase perturbations are applied which lead to small distortions of the shape of the system. As a consequence a directed current is generated [2]. The pumped current is a consequence of quantum interference effects. An elegant formulation of the problem has been achieved by Brouwer [3] based on the modulation of the emissivities of the system 何. Inelastic scattering does not suppress the pumped current but introduces an additional more classical contribution to the pumped current due to rectification [5,6]. For a broader view of this very active field we refer to a few recent works [7 [1].

Adiabatic pumping is of general interest due to the fact that only very slow perturbations are required: furthermore if the amplitudes are small the system remains at all times close to the stationary equilibrium state. Thus parametric adiabatic pumping can be viewed as a tool to investigate the near equilibrium properties of a system. Since perturbations can be applied locally such an investigation gives information on the system which can not be obtained through the application of global and stationary forces.

It is the purpose of this work to complement the quantum mechanical discussions mentioned above and to investigate parametric pumping for a purely classical system. We consider particles subject to damping and thermal noise in a symmetric periodic potential $V_{0}(x)=V_{0}(-x)$. In addition to the static potential $V_{0}(x)$ two small amplitude time-dependent oscillatory potentials act on the particles. The perturbations we consider are periodic in time and are periodic in space with the same period as the static potential. We investigate the case where the perturbations have a double phasedifference both in time and space. As in the quantum case, a directed current is generated which is proportional to the frequency of the oscillating perturbations and proportional to the product of their amplitudes. A directed current results for almost all type of perturbations, unless these perturbations have a special symmetry (phase- differences equal to a multiple of $\pi$ ). Interestingly, for the small amplitude perturbations considered here, the thermal noise is essential: the pumped current vanishes in the zero-temperature limit, is maximal at some intermediate temperature, and vanishes in the high temperature limit.

A symmetry breaking of the system is necessary to generate a directed current. In the noise assisted parametric pumping process discussed here the symmetry is broken not with the help of the static potential $V_{0}(x)$ but through the perturbations applied to the system. Nonequilibrium state-dependent noise with the same period as the potential but out of phase with a symmetric static potential also leads to a directed current [12 [16]. Thus this is an example of directed motion in a symmetric potential for which the symmetry is broken not by the static potential but only by the non-equilibrium noise. Similarly directed motion can be obtained in systems with a spatially symmetric potential but with a friction constant which is state dependent [17, 18]. We emphasize the symmetry of the static problem, since typically, the recent literature has emphasized directed transport in systems in which already the static potential [19 21] is asymmetric $V_{0}(x) \neq V_{0}(-x)$. The examples discussed here and in Refs. 12-16] demonstrate that static symmetric breaking, i. e. the consideration of a ratchet potential 22 26] is not necessary, if either non-equilibrium noise, or perturbations applied to the system act in a symmetry breaking way.

Quasi-adiabatic perturbations of particles subject to the Smoluchowski equation have recently been investigated by Parrondo [27]. Below we present a discussion of small amplitude parametric pumping which closely follows the discussion by Parrondo [27].

\section{PARAMETRIC PUMPING}

The overdamped motion of a classical particle in an external potential and subjected to thermal noise is governed by the Smoluchowski equation for the probability density $\rho(x, t)$, 


$$
\begin{aligned}
\frac{\partial}{\partial t} \rho(x, t) & =\mu \frac{\partial}{\partial x}\left[\frac{\partial V(x, \vec{T}(t))}{\partial x}+\frac{1}{\beta} \frac{\partial}{\partial x}\right] \rho(x, t) \\
& =-\frac{\partial}{\partial x} J(x, t) \rho(x, t),
\end{aligned}
$$

where $\mu$ is the mobility, $\beta$ the inverse temperature and $J(x, t)=-\mu V^{\prime}(x, \vec{T}(t))-\mu k T \partial / \partial x$ is the current operator. Here the prime denotes derivative with respect to $x$. We consider a total potential $V(x, \vec{T}(t))$ that is written as a sum of a symmetric periodic potential

$$
V_{0}(x)=V_{0}[1-\cos (2 \pi x / a)]
$$

with period $a$, plus a perturbation

$$
\Delta V(x, \vec{T}(t))=X_{1}(x) T_{1}(t)+X_{2}(x) T_{2}(t) .
$$

Here $X_{1}(x)$ and $X_{2}(x)$ are arbitrary spatial functions with period $a$ and similarly $T_{1}(t)$ and $T_{2}(t)$ are arbitrary functions of time with period $2 \pi / \omega$. We consider two special examples with purely harmonic driving. In both examples the time-dependent external perturbation is composed of two sinusoidal potentials with amplitude $V_{A}$ and $V_{B}$. In the first example the spatial functions act over the entire period,

$$
\begin{aligned}
\Delta V(x, \vec{T}(t))= & -V_{A} \cos (2 \pi x / a) \cos (\omega t) \\
& -V_{B} \cos (2 \pi x / a+\chi) \cos (\omega t+\varphi)
\end{aligned}
$$

with a phase difference $\chi$ in space and a phase difference $\varphi$ in time. In the second example driving is spatially localized at two arbitrary points $x_{1}$ and $x_{2}$ in the interval $[0, a]$

$$
\begin{aligned}
\Delta V(x, \vec{T}(t))= & -V_{A} \cos (\omega t) \delta\left(x_{1}-2 \pi x / a\right) \\
& -V_{B} \cos (\omega t+\varphi) \delta\left(x_{2}-2 \pi x / a\right)
\end{aligned}
$$

where $\delta$ is the Dirac delta function. In the following we assume that $\Delta V(x, \vec{T}(t))$ changes slowly in time and that its amplitude is small compared to the unperturbed potential $V_{A}, V_{B} \ll V_{0}$.

The quantity of prime interest is the mean particle current, averaged over one period of space and one period of time,

$$
\begin{aligned}
\langle I\rangle & =\frac{\omega}{2 \pi a} \int_{0}^{a} d x \int_{0}^{\frac{2 \pi}{\omega}} d t J(x, t) \rho(x, t) \\
& =-\frac{\mu \omega}{2 \pi a} \int_{0}^{a} d x \int_{0}^{\frac{2 \pi}{\omega}} d t V^{\prime}(x, \vec{T}(t)) \rho(x, t) .
\end{aligned}
$$

Due to the periodicity of the potential in time and space, the second term of $J(x, t)$ does not contribute to the current. We begin by solving the Smoluchowski equation (11) in the limit of small driving frequencies, $\omega \rightarrow 0$. In this limit, the system remains close to the adiabatic solution $\rho_{0}^{-}(x, t)$, which is obtained by setting in Eq. (1) $\partial \rho / \partial t=0$. The latter is given by an equilibrium Boltzmann distribution

$$
\begin{aligned}
\rho_{0}^{ \pm}(x, t) & =Z_{ \pm}^{-1}(t) e^{-\beta V(x, \vec{T}(t))} \\
Z_{ \pm}(t) & =\int_{0}^{a} d x e^{ \pm \beta V(x, \vec{T}(t))},
\end{aligned}
$$

and therefore does not yield any current. (Here we have in addition to the adiabatic solution $\rho_{0}^{-}(x, t)$ introduced $\rho_{0}^{+}(x, t)$ for later reference). Since the adiabatic solution is not associated with a current flow, we need to find the correction to this solution to determine the current. We expect that the correction to the adiabatic solution is of the order of the variation rate of the perturbation [27], which, for the potential $\Delta V(x, \vec{T}(t))$ we consider here, is given by $\omega$. We thus seek a solution of the Smoluchowski equation of the form

$$
\rho(x, t) \simeq \rho_{0}^{-}(x, t)+\dot{\vec{T}}(t) \vec{\nu}(x, \vec{T}(t)) .
$$

The correction $\dot{\vec{T}}(t) \vec{\nu}(x, \vec{T}(t))$, of order $\omega$, to the adiabatic solution $\rho_{0}^{-}(x, t)$ gives rise to the non-vanishing particle current. Inserting the ansatz (8) into the Smoluchowski equation (11) and neglecting the time derivative of the correction, which is of the order $\omega^{2}$, we arrive at

$$
\mu \frac{\partial}{\partial x}\left[\frac{\partial V(x, \vec{T}(t))}{\partial x}+\frac{1}{\beta} \frac{\partial}{\partial x}\right] \vec{\nu}(x, \vec{T}(t))=\vec{\nabla}_{\vec{T}(t)} \rho_{0}^{-}(x, t) .
$$

This second-order partial differential equation for $\vec{\nu}(x, \vec{T}(t))$ has to be solved with periodic boundary conditions, $\vec{\nu}(0, \vec{T}(t))=\vec{\nu}(a, \vec{T}(t))$, and the condition that the integral of $\vec{\nu}(x, \vec{T}(t))$ along the interval $[0, a]$ vanishes. This second condition follows from the normalization of $\rho(x, t)$ over one (spatial) period. We find

$$
\begin{aligned}
& \vec{\nu}(x, \vec{T}(t))=\vec{C}_{1} e^{-\beta V(x, \vec{T}(t))} \int_{0}^{x} d y e^{\beta V(y, \vec{T}(t))} \\
& +\frac{\beta}{\mu} e^{-\beta V(x, \vec{T}(t))} \int_{0}^{x} d y e^{\beta V(y, \vec{T}(t))} \int_{0}^{y} d z \vec{\nabla}_{\vec{T}(t)} \rho_{0}^{-}(z, t) \\
& +\vec{C}_{2} e^{-\beta V(x, \vec{T}(t))}
\end{aligned}
$$

where $\vec{C}_{1}(t)$ and $\vec{C}_{2}(t)$ are two vectors of integration constants. Explicitly, $\vec{C}_{1}(t)$ is given by

$$
\vec{C}_{1}(t)=-\frac{\beta}{\mu} \int_{0}^{a} d x \rho_{0}^{+}(x, t) \int_{0}^{x} d y \vec{\nabla}_{\vec{T}(t)} \rho_{0}^{-}(y, t),
$$


where $\rho_{0}^{ \pm}(x, t)$ is given by Eq. (7). The solution (8) of the Smoluchowski equation is then obtained by combining Eqs. (7) and (10). Using the above solution, the mean current (6) can be easily calculated,

$$
\begin{aligned}
\langle I\rangle & =-\frac{\mu \omega^{2}}{2 \pi \beta \omega_{0}} \int_{0}^{\frac{2 \pi}{\omega}} d t \dot{\vec{T}}(t) \vec{C}_{1}(t) \\
& =\frac{\omega}{2 \pi} \int_{\vec{T}(0)}^{\vec{T}\left(\frac{2 \pi}{\omega}\right)} d \vec{T} \int_{0}^{a} d x \rho_{0}^{+}(x, t) \int_{0}^{x} d y \vec{\nabla}_{\vec{T}(t)} \rho_{0}^{-}(y, t) .
\end{aligned}
$$

We now take the case $\Delta V(x, \vec{T}(t))=T_{1}(t) X_{1}(x)+$ $T_{2}(t) X_{2}(x)$. With $\vec{\nabla}_{\vec{T}(t)}=\partial / \partial T_{1}+\partial / \partial T_{2}$ and Green's theorem, Eq. (12) can be rewritten as :

$$
\begin{array}{r}
\langle I\rangle=\frac{\omega}{2 \pi} \int_{A} d T_{1} d T_{2} \int_{0}^{a} d x \int_{0}^{x} d y\left(\frac{\partial \rho_{0}^{+}(x, t)}{\partial T_{1}} \frac{\partial \rho_{0}^{-}(y, t)}{\partial T_{2}}\right. \\
\left.-\frac{\partial \rho_{0}^{+}(x, t)}{\partial T_{2}} \frac{\partial \rho_{0}^{-}(y, t)}{\partial T_{1}}\right) .
\end{array}
$$

This is the key result of our paper. Eq. 13 gives the pumped current in terms of the derivative of the adiabatic solution $\rho_{0}^{-}(x, t)$ and its companion $\rho_{0}^{+}(x, t)$. It is valid for slowly time-varying periodic potentials $\Delta V(x, \vec{T}(t))$ of arbitrary shape and arbitrary strength. The expression Eq. (13) is most useful for the case of small amplitude perturbations. In this case the derivatives of $\rho_{0}^{ \pm}(x, t)$ can be evaluated at zero amplitude and the integral over the area enclosed by the path $\int d T_{1} d T_{2}$ is simply a multiplying factor.

\section{EXAMPLE WITH GLOBAL DRIVING}

We now specialize to the small amplitude regime. To do so, we can set $T_{1}=T_{2}=0$ in the integrand of Eq. (13). For the particular potential $\Delta V(x, \vec{T}(t))$ introduced in Eq. (4) the area enclosed by the pumping path is $\int_{A} d T_{1} d T_{2} V_{A} V_{B} \sin \varphi \sin \chi$ and a calculation leads to the pumped current

$$
\langle I\rangle=\frac{1}{2 \pi V_{0}^{2}} \beta V_{0} \frac{I_{1}\left(\beta V_{0}\right)}{I_{0}^{3}\left(\beta V_{0}\right)} \omega V_{A} V_{B} \sin \varphi \sin \chi,
$$

where $I_{0}(x)$ and $I_{1}(x)$ are the hyperbolic Bessel functions of order zero and one, respectively. Equation (14) exhibits the main features of adiabatic pumping. We see that the adiabatically pumped current $\langle I\rangle$ is linear in the pumping frequency $\omega$ and the amplitudes $V_{A}$ and $V_{B}$ of the two external potentials. The current is proportional to the sines of the temporal and spatial phase differences. An important consequence of Eq. (14) is that the current vanishes if either $\varphi$ or $\chi$ is a multiple of $\pi$.
This shows that a double phase difference, both in time and in space is necessary in order to rectify the noise. More generally, it can be shown that if the perturbation is written as product $\Delta V(x, \vec{T}(t))=X(x) T(t)$ with $X(x)$ periodic in space and $T(t)$ periodic in time, there is no pumped current, for any amplitude. An interesting situation, which offers a simple physical interpretation, is the one for which the current is maximum. This happens when $\varphi, \chi= \pm \frac{\pi}{2}$. By taking $\left|V_{A}\right|=\left|V_{B}\right|$, the perturbation can be rewritten in the form

$$
\Delta V(x, \vec{T}(t))=V_{A} \cos (2 \pi x / a \pm \omega t),
$$

the sign being determined by the relation between $\varphi$ and $\chi$ and between $V_{A}$ and $V_{B}$. The maximum current is hence generated by a traveling wave potential. It is to be expected that a traveling wave potential is a particularly efficient way of generating a current [28,29].

Let us now examine the temperature dependence of the particle current. It is given by the function

$$
f_{\text {pump }}\left(u=\beta V_{0}\right)=u \frac{I_{1}(u)}{I_{0}^{3}(u)} .
$$

This function has been plotted in Fig.(11) (solid line). We observe that $f_{\text {pump }}(u)$ vanishes both in the limit of low and high temperature and that it reaches a maximum at $u \simeq 1.426 \ldots$ This behavior can be understood as follows. At very low temperature (large $u$ ), thermal activation is negligible. Since the perturbation $\Delta V(x, \vec{T}(t))$ is furthermore very small, the particle remains trapped in the minima of the bare potential $V_{0}(x)$ and there is no transport. For the small amplitude perturbations considered here this clearly demonstrates that there is no classical pumping without thermal noise. In the opposite limit of high temperature (small $u$ ), thermal fluctuations dominate and we have simple (symmetric) Brownian diffusion with zero average displacement. The maximum pumped current corresponds to a thermal energy of the order of the potential energy.

It is further instructive to compare the adiabatic pumped current (14) with the current created by a small constant (time and space independent) external force $F$. In this case the overdamped Brownian particle experiences the potential, $V(x)=V_{0}(x)-F x$. A similar calculation of the current, up to first order in $F$, yields the following expression (see also [30]),

$$
\langle I\rangle=\frac{\mu F}{a I_{0}^{2}\left(\beta V_{0}\right)} .
$$

The temperature dependence of this force induced current, given by $f_{\text {force }}(u)=I_{0}^{-2}(u)$, is shown in Fig.(11) (dashed line). Here, in contrast to adiabatic pumping, the current is maximum at very high temperature. This is due to the presence of a small but finite slope in the potential. In the low temperature limit both currents are suppressed. 


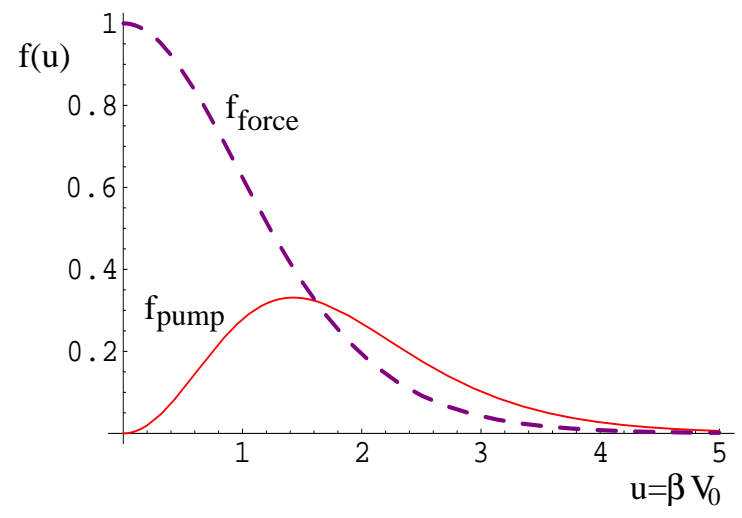

FIG. 1. Temperature dependence $f\left(u=\beta V_{0}\right)$, of the adiabatically pumped current (14) (solid line) and the current (17) generated by a constant external force $F$ (dashed line).

We notice, however, that for the force induced current, $f_{\text {force }}(u) \sim u e^{-2 u}$, the decay with temperature is asymptotically faster than for the pumped current, $f_{\text {pump }}(u) \sim u^{2} e^{-2 u}$. As a consequence, there is a critical temperature $\beta_{c}$ below which the pumped current is larger than the current generated by $F$. In this regime, adiabatic pumping is a more efficient transport mechanism than applying an external force. The value of the critical temperature $\beta_{c}$ depends on both the area enclosed by the pumping path, the frequency and $F$.

\section{EXAMPLE WITH LOCALIZED DRIVING}

In the example discussed above the perturbation potential is extended through the entire spatial period of the system. Is this a necessary condition, or is a spatially localized perturbation sufficient to generate a pumping current? To answer this question we consider a perturbation given by Eq. (5). As for the previous example, we can compute the current from Eq. (13) to obtain:

$$
\langle I\rangle=-\frac{\omega V_{A} V_{B} \sin \varphi}{8 \pi^{3} V_{0}^{2}}\left(\frac{\beta V_{0}}{I_{0}\left(\beta V_{0}\right)}\right)^{2} h\left(\beta V_{0}, x_{1}, x_{2}\right),
$$

where $h\left(\beta V_{0}, x_{1}, x_{2}\right)=f\left(\beta V_{0}, x_{1}, x_{2}\right)+f\left(-\beta V_{0}, x_{1}, x_{2}\right)$, and

$$
\begin{aligned}
f\left(\beta V_{0}, x_{1}, x_{2}\right)= & e^{\left(2 \beta V_{0} \sin \left(x_{1}+x_{2}\right) \sin \left(x_{1}-x_{2}\right)\right)} \\
& \times\left(\theta\left(x_{1}-x_{2}\right)+\int_{x_{1}}^{x_{2}-\pi} d x \tilde{\rho}_{0}^{-}(x)\right),
\end{aligned}
$$

with $\tilde{\rho}_{0}^{-}(x)=\rho_{0}^{-}\left(x, T_{1}=0, T_{2}=0\right)$. $\theta$ is the Heaviside step function. If we exchange $x_{1}$ and $x_{2}$, we change the sign of the current, since it corresponds to a change of the sign of the temporal phase difference. The temperature dependence is similar to the one encountered previously (i.e. the current vanishes for both zero and infinite $\left.\beta V_{0}\right)$. The interesting dependence of the current on $x_{1}$ and $x_{2}$ is determined by $h\left(\beta V_{0}=1, x_{1}, x_{2}\right)$ which is plotted in Fig.(2). No current is generated if $x_{1}=x_{2}$. The pumped current (the function $\left.h\left(\beta V_{0}, x_{1}, x_{2}\right)\right)$ is discontinuous along the line $x_{1}=x_{2}$. Of course this discontinuity disappears if instead of a delta functions slightly broadened functions are used to describe the perturbation.

The reason that the localized perturbation leads to a pumped current is due to the normalization of the distribution function. As a consequence even a localized perturbation generates a non-local response.

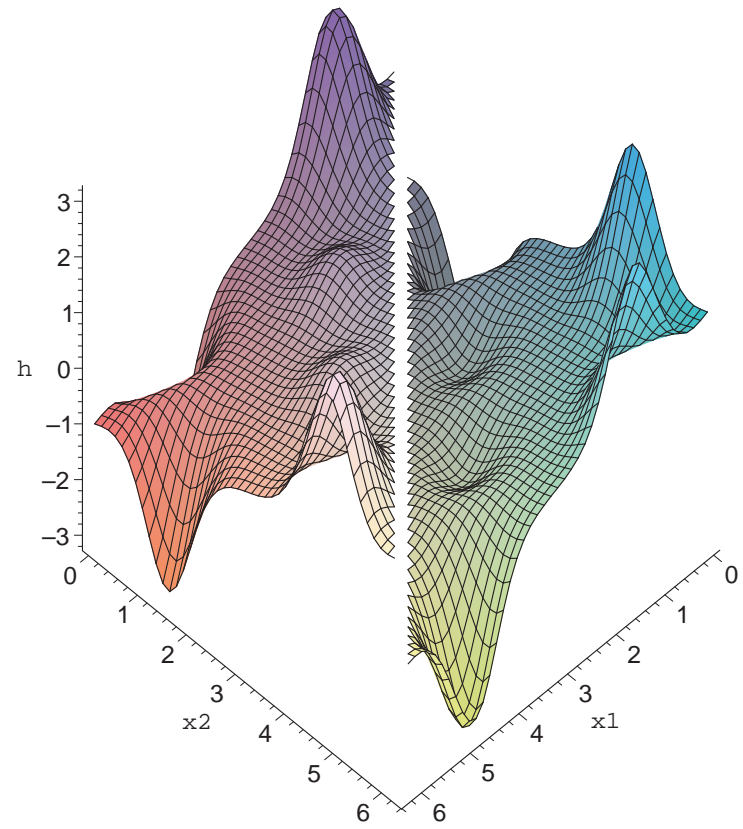

FIG. 2. $h\left(1, x_{1}, x_{2}\right)$, which describes the variation of the pumped current with respect to the positions $x_{1}$ and $x_{2}$ of the delta perturbations. There is no pumped current for $x_{1}=x_{2}$.

Fig. (2) gives an overview of the pumping currents that can be achieved with the two localized perturbations. To gain further insight it is useful to consider several cuts through Fig. (2).

In Fig. (3) (a) we keep the position of one perturbation at a fixed location $x_{2}=0$ (at the potential minimum) and consider the pumping current as a function of the position $x_{1}$ of the other perturbation. The pumped current is then maximal if $x_{1}$ is at the inflection point of the potential $\left(x_{1}=\pi / 2\right)$. There is no current if the perturbation is located at the maximum of the potential. As $x_{1}$ increases further the current direction is reversed. As $x_{1}$ passes through zero the current jumps from a negative value to a positive value. Note that in this case the pumped current is anti-symmetric around $x_{1}=\pi$ since one perturbation is located at a symmetry point $\left(x_{2}=0\right)$ of the potential. 

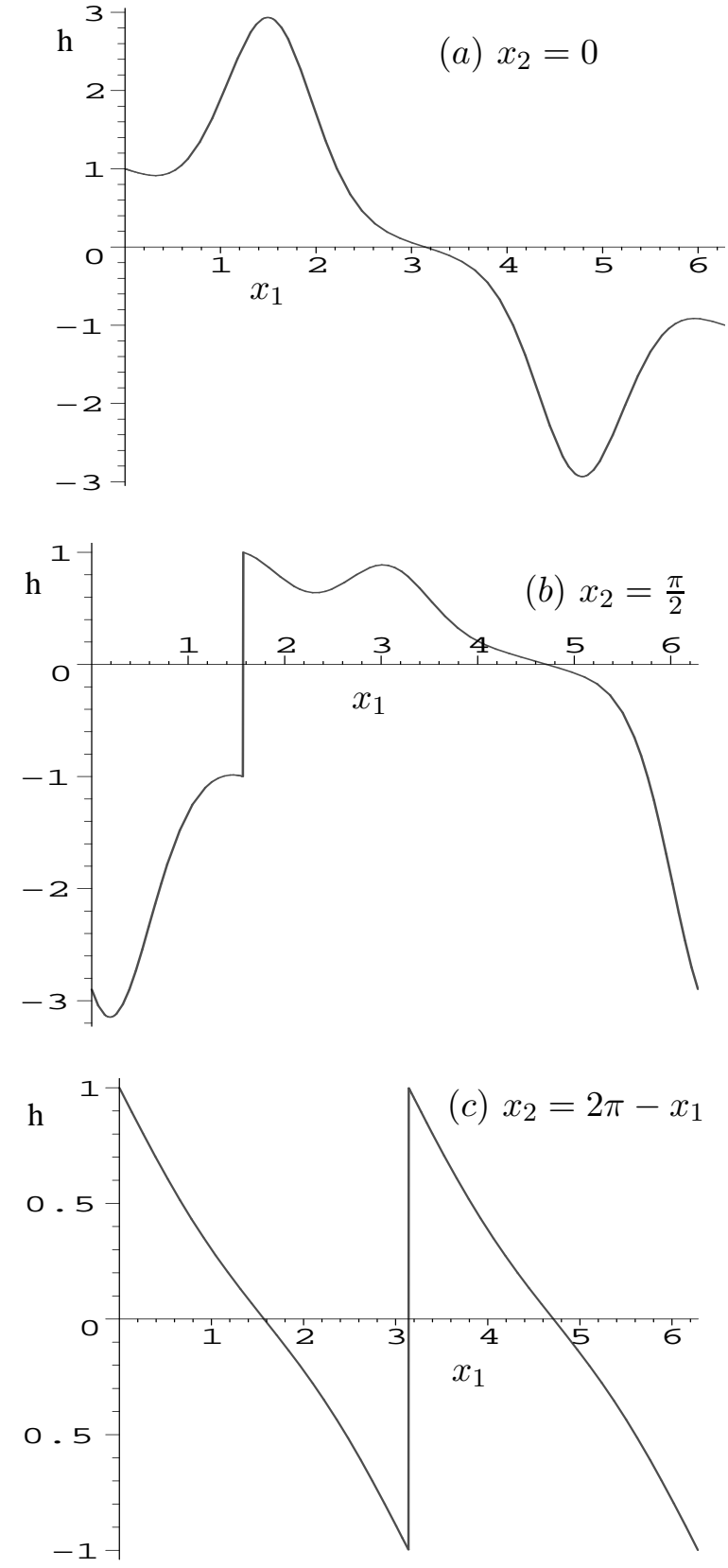

FIG. 3. Variation of the pumped current of the localized parametric pumping model. Shown is the function $h$ for (a) $x_{2}=0$, (b) $x_{2}=\frac{\pi}{2}$ and (c) $x_{2}=2 \pi-x_{1}$.

In Fig. (3) (b) we keep one perturbation at the inflection point of the potential $x_{2}=\pi / 2$. As a function of $x_{1}$ the pumped current jumps at $x_{1}=\pi / 2$. The current decreases as $x_{1}$ increases past $x_{2}=\pi / 2$ goes through a local minimum, reaches a local maximum for $x_{1}$ just before $x_{1}=\pi$. The maximal pumped current is achieved if $x_{1}$ is a little to the right of the local potential minimum. Note that in this case we have no symmetry around $\pi / 2$ and neither are the two directions of current equivalent.

In Fig. (3) (c) we consider the case where the two perturbations are symmetrically located around the potential maximum $\pi$. The current then jumps at $x_{1}=x_{2}=\pi$ and is antisymmetric as a function of $x_{1}$ around this point.

For global driving we found that the current is a sinusoidal function of the spatial phase difference between the two perturbations. In contrast, in the local driving model considered here the pumped current depends not in a simple manner only on the spatial distance between the two perturbations but also on their absolute locations in the interval. This leads to the much more complicated behavior depicted Fig. (2) and Fig. (3) (b).

\section{DISCUSSION}

We first point to the remarkable fact that the pumped current is independent of the mobility $\mu$. This should be contrasted with the linear dependence on $\mu$ of a current generated with the help of a constant force. That the pumped current is indeed independent of $\mu$ can easily be seen by considering Eq. (9). The solution of this equation determines the correction $\nu$ of the density to the adiabatic solution: This correction is proportional to $\mu^{-1}$. Since the current operator is proportional to $\mu$ the resulting net current is independent of $\mu$. We emphasize that the pumped current is independent of $\mu$ not only for the particular models considered here but quite generally for all adiabatic perturbations.

In summary, we have shown that a directed current can be generated in a symmetric periodic potential by adiabatically modulating two small external potential parameters. We have investigated a model with global (spatially periodic) perturbations and a model with localized perturbations. For the model with global perturbations we find that a double - temporal and spatial - phase difference is necessary to generate a current. The maximum pumped current is obtained for a temperature $k T$ of the order of the potential height $V_{0}$ and for a perturbative potential corresponding to a traveling wave $(\varphi, \chi= \pm \pi / 2)$. Similarly for the model with localized perturbations we find a pumped current unless the two perturbations are located at the same position or have a spatial difference of $\pi$. Pumping arises through the subtle interplay between thermal fluctuations and cyclic variations of the potential. It therefore disappears in the limit of low and high temperature when either the potential or the thermal energy becomes predominant. We have demonstrated the existence of a potential dependent critical temperature below which adiabatic pumping is a more efficient than applying a small constant external force. The work presented here can be extended in different directions: Systems with open boundary conditions, several space dimensions and inertial effects are possible subjects for further research.

We thank M. Moskalets and A. Alekseev for discussions. This work was supported by the RTD network Nanoscale Dynamics, Coherence and Computation and by the Swiss National Science Foundation. 
[1] M. Switkes, C. H. Marcus, K. Campman and A. C. Gossard Science 283, 1905 (1999).

[2] F. Zhou, B. Spivak, and B. Altshuler, Phys. Rev. Lett. 82, 608 (1999).

[3] P. W. Brouwer, Phys. Rev. B 58, R10135 (1998).

[4] M. Büttiker, H. Thomas, and A. Prêtre, Z. Phys. B 94, 133 (1994).

[5] M. Moskalets and M. Büttiker, Phys. Rev. B 64, 201305(R) (2001).

[6] J. N. H. J. Cremers and P. W. Brouwer, Phys. Rev. B 65, 115333 (2002).

[7] J. E. Avron, A. Elgart, G. M. Graf, and L. Sadun, Phys. Rev. B 62, R10618 (2000); J. E. Avron, A. Elgart, G. M. Graf and L. Sadun, Phys. Rev. Lett. 87, 236601 (2001).

[8] A. Alekseev, (unpublished). cond-mat/0201474

[9] M. Moskalets and M. Büttiker, Phys. Rev. B (unpublished). cond-mat/0201259

[10] M. L. Polianski, M. G. Vavilov, P. W. Brouwer, (unpublished). cond-mat/0202241

[11] B. G. Wang and J. Wang, (unpublished). condmat/0204067, B. G. Wang, J. Wang, and H. Guo, Phys. Rev. B 65, 073306 (2002).

[12] M. Büttiker, Z. Phys. B 68, 161 (1987).

[13] N. G. van Kampen, IBM J. Res. Develop. 32, 107 (1988).

[14] R. Landauer, J. Stat. Phys. 53, 233 (1988).

[15] Ya. M. Blanter and M. Büttiker, Phys. Rev. Lett. 81, 4040 (1998).

[16] T. Hondou and K. Sekimoto, Phys. Rev. E 62, 6021 (2000); K. Sekimoto, F. Takagi, T. Hondou, Phys. Rev. E 62, 7759 (2000).

[17] R. H. Luchsinger, Phys. Rev. E62, 272 (2000).

[18] D. Dan, M. C. Mahato, and A. M. Jayannavar, Physica A 296, 375 (2001).

[19] F. Jülicher, A. Adjari, and J. Prost, Rev. Mod. Phys. 69, 1269 (1997).

[20] R. D. Astumian, Science 276, 917 (1997).

[21] P. Reimann, Phys. Rep. 361, 57 (2002).

[22] M. O. Magnasco, Phys. Rev. Lett. 71, 1477 (1993); M. O. Magnasco and G. Stolovitzky, J. Stat. Phys. 93, 615 (1998).

[23] R. D. Astumian and M. Bier, Phys. Rev. Lett. 72, 1766 (1994).

[24] M. M. Millonas and D. I. Dykman, Phys. Lett. A 183, 65 (1994).

[25] C. R. Doering, W. Horsthemke, and J. Riordan, Phys. Rev. Lett. 72, 2984 (1994).

[26] J. Luczka, R. Bartussek and P. Hanggi, Europhys. Lett. 31, 431 (1995); J. H. Li, J. Luczka, P. Hanggi, Phys. Rev. E64, 011113 (2001).

[27] J. M. Parrondo, Phys. Rev. E 57, 7297 (1998).

[28] Brownian motion in a traveling wave potential in the absence of a static periodic potential can be mapped on the problem of a Brownian motion in a static tilted periodic potential. See: R. Landauer and M. Büttiker, Phys. Scr. T9, 155 (1985); L. P. Faucheux, G. Stolovitzky, and A.
Libchaber, Phys. Rev. E 51, 5239 (1995); M. Borromeo and F. Marchesoni, Phys. Lett. A 249, 8457 (1998).

[29] In mesoscopic electrical transport surface accoustic waves can be used to generate traveling wave potentials: J. M. Shilton, D. R. Mace, V. I. Talyanskii, Yu. Galperin, M. Y. Simmons, M. Pepper, and D.A. Ritchie, J. Phys.: Condens. Matter 8, L337 (1996); V. I. Talyanskii, J. M. Shilton, M. Pepper, C. G. Smith, C. J. B. Ford, E. H. Linfield, D. A. Ritchie, and G. A. C. Jones, Phys. Rev. B 56, 15180 (1997); O. Entin-Wohlman, Y. Levinson, and P. Wölfle, Phys. Rev. B 64, 195308 (2001).

[30] H. Risken, The Fokker-Planck Equation (Springer, Berlin, 1989). 